\title{
Spontaneous cervical epidural hematoma: case report
}

\author{
Adnan Awada ${ }^{1}$, Neville Russell ${ }^{2}$, Naif Al Fayez ${ }^{2}$, Richard Naufal ${ }^{3}$ and Hussein Al Kohlani ${ }^{2}$ \\ Sections of ${ }^{1}$ Neurology and ${ }^{2}$ Neurosurgery and ${ }^{3}$ Department of Medical Imaging, King Fahd National Guard \\ Hospital, POB 22490, Riyadh 11426, Saudi Arabia
}

\begin{abstract}
Spontaneous spinal epidural hematoma (SSEH) is an uncommon cause of acute nontraumatic myelopathy. We report a 14 year-old boy who had tetraplegia on awakening. Diagnosis of spinal epidural hematoma was made by magnetic resonance imaging. Despite spinal cord decompression within $9 \mathrm{~h}$ from onset, he remained tetraplegic. No cause for the bleeding was found. The pathogenic hypotheses of SSEH are discussed and the importance of rapid diagnosis and treatment is emphasized.
\end{abstract}

Keywords: spinal cord; epidural hematoma; magnetic resonance imaging (MRI); acute myelopathy; tetraplegia

\section{Introduction}

Spinal epidural hematoma is a very rare cause of acute spinal cord compression. It is important to recognize it since early diagnosis and prompt surgical evacuation provide the maximum chance of functional recovery. Spinal epidural hematoma has been reported in association with blood dyscrasias, coagulopathies, anti-coagulant treatment, infection, tumor, pregnancy and vascular malformations, but approximately half of the cases have no apparent cause.

The cervical variety of spinal epidural hematoma is usually spontaneous and of acute onset ${ }^{2}$ while hematomas occurring at lower levels of the spine have a more subacute or chronic course. Moreover, in the latter, the cause of the bleeding is more likely to be defined. $^{3,4}$

The case reported here illustrates this rare entity and gives us the opportunity to comment on some of the features of spontaneous cervical epidural hematomas.

\section{Case report}

This 14 year-old boy awakened on the 3rd of July 1993 at 9 am with severe neck pain and difficulty in moving all four limbs. Idiopathic epilepsy had been diagnosed when he was 12 years old and was being treated with carbamazepine $200 \mathrm{mg}$ three times daily. He had had no seizures in the preceding month and had not recently participated in any form of athletic activity. On examination in the emergency room at approximately 12 noon, he was tetraplegic with a motor and sensory level at C5-C6. The deep tendon reflexes were absent and there was no response to plantar

Correspondence: Dr A Awada stimulation. Urinary retention and priapism were present. An acute cervical spinal cord disorder was suspected and an urgent magnetic resonance imaging (MRI) examination ordered. This was performed at $3 \mathrm{pm}$ and on T1 and T2-weighted images, revealed an iso-intense mass, centered at the level of C6 (a 'double cord' image) (Figure 1). This was compressing the dorsal aspect of the spinal cord. Three hours later a C4-C7 laminectomy was performed. The posterior epidural space contained a large semisolid clot. After its removal the dura-enclosed spinal cord appeared to re-expand and pulsation returned. It was not necessary to extend the laminectomy caudal to $\mathrm{C} 7$, because at this level the hematoma was liquid and spontaneously flowed into the decompression site. No vascular malformation or any bleeding source was found. A

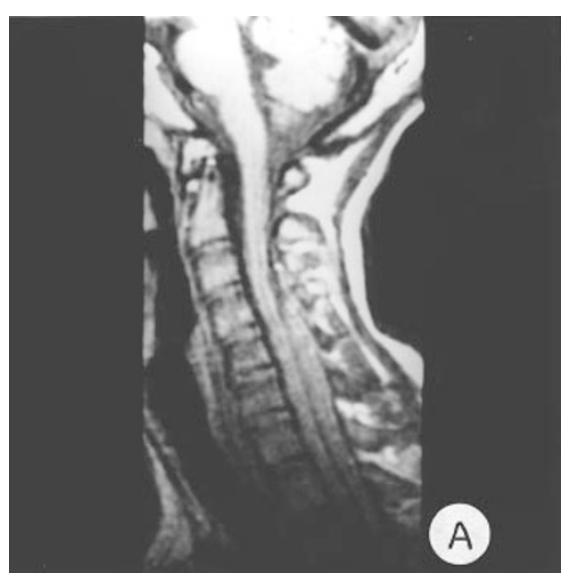

Figure 1 T1-weighted saggital magnetic resonance image: Iso-intense mass compressing the posterior aspect of the spinal cord from C2 to T2 ('double cord' image) 
complete blood count and a coagulation profile were normal.

Post operative improvement was minimal and after 3 years, he remains wheel chair bound, with a sensorimotor level at C7-C8. A subsequent MRI scan did not show any image suggestive of a vascular malformation.

\section{Discussion}

Up to 1984, only 43 cases of acute spontaneous cervical epidural hematoma (ASCEH) had been reported. ${ }^{2}$ Although the source of bleeding has never been clarified, it has usually been assumed to be of venous origin. In an extensive review published in the Handbook of Clinical Neurology in 1976, ${ }^{1}$ Bruyn and Bosma theorized that spontaneous spinal epidural hematomas occur by the following mechanism: there is local pooling within valveless, thin-walled epidural veins and brief increases in intravenous pressure, both intra-thoracic and intra-abdominal, may lead to their rupture. This might account for those cases reported to have occurred in association with voiding, bending, turning in bed at night, straining at stool, sneezing and coitus. ${ }^{5}$ While this explanation may be valid for the subacute or chronic hematomas of the lumbar region, it is unsatisfactory to explain ASCEH. Venous pressure is low in the epidural veins of the cervical region even lower than the intrathecal pressure. $^{2}$ Also the normally expanded dural sac can easily tamponade epidural venous bleeding as is routinely demonstrated during spinal surgery. ${ }^{2}$ In addition the rapidity with which spinal cord compression develops tends to favor arterial bleeding. After analyzing the anatomy of the blood supply of the cervical spine, Beatty and Winston postulated that the source of bleeding was the 'free' anastomotic arteries that run in the epidural space and connect with the radicular arteries. ${ }^{2}$ Since $90 \%$ of ASCEH are located in the $\mathrm{C} 6-\mathrm{C} 7$ region, a highly mobile segment of the spine, they believed that certain movements at this level might stretch the free arteries beyond their limits of tolerance, causing them to rupture. The location of the hematoma in our case, the rapidity of progression of the spinal cord compression and the operative findings tend to support a hypothesis of arterial bleeding.

The clinical features of spinal epidural hematoma are stereotyped. Symptoms of sudden severe spinal pain followed by a progressive paraplegia or tetraplegia should suggest the diagnosis. Magnetic resonance imaging (MRI) scan is the investigation of choice for suspected ASCEH. ${ }^{6}$ However, fresh blood is usually iso-intense on $\mathrm{T} 1$ and $\mathrm{T} 2$ weighted images and may be difficult to interpret. An image of 'double cord' as demonstrated in our case is highly suggestive of the diagnosis. If MRI is unavailable, computerized tomographic (CT) scanning may demonstrate the blood and CT myelography will show blockage of the contrast flow.

The prognosis appears to be related to the severity of the pre-operative neurologic deficit and early operation for rapid decompression of the spinal cord is crucial. ${ }^{7}$ In a review of thirty cases of spinal epidural hematoma Lawton et $a l^{8}$ confirmed the relationship between neurological recovery, the timing of surgery and the pre-operative neurological status. They showed functional recovery to correlate inversely with the duration of spinal cord compression and the degree of severity. In addition, six of their eight patients with complete loss of neurological function improved after surgery. This led them to suggest that, despite evidence that pre-operative neurological status generally predicts outcome, surgery is not absolutely contraindicated in patients with maximum deficit. They found support for this thesis in the work of Harrison and $\mathrm{McDonald}^{9}$ whose experiments on spinal cord compression in cats showed that recovery occurred when the injury had caused demyelination of the spinal cord white matter rather than axonal disruption.

Our patient's symptoms evolved rapidly and by the time of surgical decompression, $9 \mathrm{~h}$ after onset, he was completely tetraplegic. There was no post-operative improvement. There is evidence to suggest that functional recovery might have been better if the pre-operative deficit was less severe. An awareness of the symptoms associated with ASCED and the pattern of their evolution is vital, if the hematoma is to be recognized and removed before irreversible cord damage has occurred.

\section{References}

1 Bruyn GW, Bosma NJ. Spinal extradural hematoma. In: Vinken PJ, Bruyn GW (eds). Handbook of Clinical Neurology, Volume 26 Amsterdam, 1976: pp 1-30.

2 Beatty RM, Winston KR. Spontaneous cervical epidural hematoma. A consideration of etiology. J Neurosurg 1984; 61: $143-148$.

3 Levitan LH, Wiens CW. Chronic lumbar extradural hematoma. CT findings. Radiology 1983; 148: $707-708$.

4 Nehls DG, Shetter AG, Hodak JA, Waggener JD. Chronic spinal epidural hematoma presenting as lumbar stenosis. Neurosurgery 1984; 14: $230-233$.

5 Markham JW, Lynge HN, Stahlman GEB. The syndrome of spontaneous spinal epidural hematoma. Report of 3 cases. $J$ Neurosurg 1967; 26: $334-342$.

6 Avrahami E et al. MRI demonstration of spontaneous acute epidural hematoma of the thoracic spine. Neuroradiology 1989; 31: $89-92$.

7 Costabile G, Husag L, Probst C. Spinal epidural hematoma. Surg Neurol 1984; 21: $489-492$.

8 Lawton MT et al. Surgical management of spinal epidural hematomas: relationship between surgical timing and neurological outcome. J Neurosurg 1995; 83: $1-7$.

9 Harrison BM, McDonald WI. Remyelination after transient experimental compression of the spinal cord. Ann Neurol 1977; 1: $542-551$. 